\title{
AN UNUSUAL COMPLICATION OF CLOSED-CHEST CARDIAC MASSAGE
}

\author{
I. E. Plrkis, M.B.B.S., F.F.A.R.C.S., AND R. N. Mahalir, M.D.*
}

THE SIMPLICITY and ease with which an effective circulation can be re-established after cardiac arrest by the closed chest method is obvious to all those who have practised the method. Since the original description of the technique by Kouwenhoven, Jude, and Knickerbocker in $1960,{ }^{1}$ and the subsequent circulation of a film made at the Johns Hopkins Hospital, ${ }^{2}$ the method has become widely known and is being practised with increasing frequency, and in some instances, lay personnel are being instructed to use this technique in the first aid treatment of the apparently dead.

Baringer et al. ${ }^{3}$ and Morgan ${ }^{4}$ have drawn attention to the complications of this method, and an editorial in the New England Journal of Medicine ${ }^{5}$ has warned against indiscriminate use of closed chest massage.

The most frequent complication is that of rib fracture, which usually has little effect on the ultimate outcome for the patient. However, multiple rib fractures can occur, and may, in spite of intensive efforts, contribute to the death of the patient. The following report illustrates such a case.

A 56-year-old housewife, admitted to the Victoria General Hospital for investigation of asthma and iridocyclitis of four years' duration, underwent bronchoscopy under local anaesthesia, to rule out the possibility of a bronchial adenoma.

During the night following the bronchoscopy she developed increasing respiratory distress, which was at first thought to be due to an exacerbation of her asthma, and treated by giving additional oxygen by mask. Her condition continued to deteriorate, and the anaesthetist was asked to see her prior to a projected tracheostomy for suspected upper respiratory tract obstruction.

The patient was orthopnoeic, semicomatose, and moderately cyanosed on oxygen. There was marked indrawing of the supracostal and intercostal spaces, and a high-pitched stridor could be heard on auscultation of the trachea. A diagnosis of upper respiratory obstruction due to laryngeal oedema was made, and it was decided to introduce an endotracheal tube to relieve the obstruction, in order to improve the patient before elective tracheostomy under general anaesthesia.

After oxygenation by positive pressure ventilation, an endotracheal tube was passed through the nostril, and the cords were visualized by a laryngoscope passed orally. There was no evidence of laryngeal oedema. The tube passed freely beyond the cords to about the level of the cricoid, where it encountered a resistance. Gentle pressure on the tube resulted in complete respiratory obstruction. The

${ }^{*}$ From the Departments of Anaesthesia and Medicine, Dalhousie University, and Victoria General Hospital, Halifax, Nova Scotia.

Can. Anaes. Soc. J., vol. 9, no. 3, May, 1962 
tube was withdrawn but complete obstruction remained, and could not be overcome with firm pressure. Emergency tracheotomy was performed under difficulties of venous engorgement and movement of the now-unconscious asphyxiated patient, and was complete in less than three minutes; but when the airway was established, the patient gave one gasp and became apnoeic. No pulse or blood pressure could be detected and massive pulmonary oedema prevented auscultation of the heart. The pupils had previously been dilated with atropine. The patient was ventilated with positive pressure oxygen via the tracheostomy while closed chest cardiac massage was carried out vigorously by three operators using this method for the first time, and working in relays for 30 minutes. No pulse could be felt, but the patient rapidly became pink. with a good capillary refill. A Noradrenaline infusion was started, and shortly afterwards the blood pressure became recordable, and was maintained at $100 \mathrm{~mm}$. Hg by adjusting the rate of the Noradrenaline drip. E.K.G. tracings 25 minutes after the arrest showed low-voltage Q.R.S. complexes with periods of arrest, but rapidly returned to normal with the restoration of the blood pressure. Gasping respirations began 10 minutes after the arrest, and became regular 15 minutes later.

With the return of spontaneous respiration, severe paradoxical movement of the chest was noted, and, on examination, it was evident that the sternum and many ribs had been fractured during the massage, resulting in a stove-in chest.

The patient made a good recovery from her cardiac arrest, consciousness returning after a further 30 minutes, with no subsequent evidence of cerebral oedema. The blood pressure remained adequate with a slow vasopressor drip. However, because of the stove-in chest, respiration was only adequate when ventilation was assisted, so that the patient was maintained on a Bennett ventilator. Increasing bronchospasm was noted throughout the night, and by next morning even controlled respiration at high pressure would not ventilate the patient sufficiently to abolish spontaneous respiration and paradox, and the left lung became collapsed.

Under light general anaesthesia, bronchoscopy was carried out, the tracheotony was revised with the insertion of a cuffed tracheostomy tube, and the sternum was stabilized by the insertion of towel clip traction through the costal cartilages. Bronchoscopy showed that the mechanical collapse of the chest wall superimposed on the mucosal oedema due to asthma had resulted in complete occlusion of the lumen of the left main bronchus, and diminution in the size of all divisions of the bronchial tree.

The patient was transferred to an Engstron respirator using positive pressures of $35 \mathrm{~mm}$. $\mathrm{Hg}$ and in spite of marked bronchospasm, ventilation was maintained at an adequate level.

The only effective agent for controlling the bronchospasm proved to be a fastrunning A.C.T.H. drip, but after 12 hours, it was thought advisable to withhold A.C.T.H. for a period, and aminophylline was isubstituted. There was an immediate recurrence of severe bronchospasm, with disappearance of the pulse and blood pressure, and. an E.K.G. monitor showed that cardiac arrest had recurred. An external pacemaker produced a transient return of cardiac action, 
but the blood pressure continued to fall in spite of vasopressors, and the patient died about 30 minutes later, some 36 hours after the original arrest.

Post mortem showed a fractured sternum, fractures of the second, third, fourth, fifth, and sixth ribs on the left, both anteriorly and posteriorly, and of the fifth and sixth ribs on the right. There was atelectasis of the right lung, and congestion of the lower lobe of the left lung. The cause of the sudden obstruction to respiration leading to the first arrest was evident in the presence of a mucosal tear 2.5 $\mathrm{cm}$. below the vocal cords, with an attached mucosal flap $1 \mathrm{~cm} . \times 1 \mathrm{~cm}$., capable of producing a valve-like obstruction of the trachea.

While the inmediate cause of deterioration in the patient's condition was the sudden recurrence of asthmatic bronchospasm, the superimposed narrowing of the bronchial tree caused by the collapse of the chest wall undoubtedly was a major factor in producing the anoxia that led to the second and fatal cardiau arrest.

\section{SIMMARY}

Successiul resuscitation from an anoxia cardiac arrest in an asthmatic patient by closed chest cardiac massage was followed by a stove-in-chest syndrome. It is considered that this complication contributed to the death of the patient 36 hours later.

\section{REFERENCES}

1. Kotwenhoven, W. B.; JUde, J. R.; \& KNickerbocker, G. G. J.A.M.A. 173: 1064 (1960). 2. Jude, J. R. Film "External Cardiac Massage" (1961). Distributed b. Smith Kline \& French Inter-American Corp., 300 Laurentian Blvd. Montreal, P.Q.

3. Baringer, J. R.; Salzman, E. W.; Jones, W. A.; \& Friedligh, A. L. New Eng. J. Med. 265: $62(1961)$

4. Morgan, R. R. New Eng. J. Med. 265: 82 (1961).

5 Editorial, New Eng. J. Med. Q560: 97 (1961). 